\title{
Inguinal Neuritis in Open Recurrent Hernia Repair
}

\author{
Robert Charles Wright, Robert James Wright \\ Cascade Hernia \& Heartburn Institute, Meridian Surgical Center, Puyallup, USA \\ Email: Bob@robertwrightmd.com
}

Received 6 May 2014; revised 4 June 2014; accepted 3 July 2014

Copyright (C) 2014 by authors and Scientific Research Publishing Inc.

This work is licensed under the Creative Commons Attribution International License (CC BY).

http://creativecommons.org/licenses/by/4.0/

(c) (i) Open Access

\begin{abstract}
PURPOSE: To investigate the pattern of occurrence of inguinal neuritis in recurrent inguinal hernia. We hypothesize that neuritis will occur in more nerves with a wider distribution than in primary repair. METHODS: Retrospective chart review of thirty consecutive recurrent inguinal hernia repairs concentrating on the occurrence of inguinal neuritis. These are not chronic pain patients. Nerves suspected of containing inguinal neuritis were sent for histologic examination. Ilioinguinal nerves were routinely resected. Operative parameters and nerve pathology reports were reviewed. These data were compared with a recent series of one hundred consecutive primary inguinal hernia repairs with a $34 \%$ incidence of inguinal neuritis. An independent statistician from Whitman University reviewed the data. RESULTS: Twenty patients were found to have inguinal neuritis among thirty recurrent open inguinal hernia repairs (66\%). This compares to $34 \%$ among primary repairs, but it is a similar rate $(P>0.42)$ assuming that the damaged nerve was left intact in $34 \%$ of these recurrences during the primary repair. In recurrent inguinal hernia $69 \%$ of neuritis occurred in the ilioinguinal nerve compared to $88 \%$ of damaged ilioinguinal nerves in the primary hernia. A test for the difference in proportions gives $P>0.10$. The most common site of neuritis occurrence in recurrent hernias with nerve damage to the ilioinguinal nerve was at the external oblique neuroperforatum among $70 \%$ of patients compared to $83 \%$ in primary cases. A test for difference in proportions gives $P>0.36$. Two separate nerves were found to exhibit neuritis in six patients $(20 \%)$ significantly higher than $1 \%$ among primary hernias $(P<0.01)$. CONCLUSION: The overall incidence of inguinal neuritis was $66 \%$ in recurrent inguinal hernia repairs. The ilioinguinal nerve was most commonly affected in these recurrent hernias. Inguinal neuritis occurs more commonly in recurrent hernia compared with primary inguinal hernia; however, it has a similar distribution. Neuritis occurs in two nerves with $20 \%$ frequency $(P<0.01)$, so all nerves should be assessed during recurrent herniorrhaphy. The data support the hypothesis.
\end{abstract}

\section{Keywords}

Hernias, Recurrent, Nerve, Inflammation, Neuritis 


\section{Introduction}

Inguinal neuritis is a degenerative condition of the named sensory nerves of the inguinal canal as found during inguinal hernia repair. The nerve is grossly noted to be twice the normal diameter of the contiguous nerve. A white thickening of the nerve typically about $1 \mathrm{~cm}$ in length is seen, possibly longer [1]. Frequently this is associated with obvious surrounding fibrosis. Neuritis is commonly found situated where the nerve pierces through the external oblique fascia. Typically this occurs at the external inguinal ring, but it may be through an "aponeurotic buttonhole" medial to the external inguinal ring (present in $10 \%$ - 18\% of patients) [2]-[4]. It is postulated that the local compressive force of the hernia damages the nerve with coughing and valsalva maneuvers. Histologically this is associated with perineural fibrosis, Renaut bodies, fibrotic epineural tissue, edema, fragmentation, and other mixed neurological findings.

Inguinal neuritis has recently been reported in primary inguinal hernias and therefore seems to be independent of prior operative trauma. Inguinal neuritis occurs in 34\% of patients with primary inguinal hernia [1]. When present it affects the ilioinguinal nerve in $88 \%$ of cases. It most frequently occurs at the neuroperforatum. At this site the nerve penetrates the fascia. Friction occurs easily at that site with the pressure of the hernia contents behind it.

Since inguinal neuritis has been shown to be present in $34 \%$ of primary inguinal patients and if operative trauma sometimes results in chronic pain [5] we postulate that inguinal neuritis will be found in more nerves and in a wider distribution in the length of that nerve in recurrent hernia than is present in primary herniorrhaphy.

\section{Methods}

A retrospective chart review was conducted of thirty consecutive recurrent inguinal hernia patients, who underwent Lichtenstein repair. The study was previously exempted by the local investigative review board. The gross appearance of nerves, visualization of the iliohypogastric, ilioinguinal and genital branch of the genitofemoral nerves were gleaned from operative reports. Neuritis was recognized by whitish nerve thickening of twice or more of the normal nerve diameter. The involved nerve and the site of involvement were determined from the dictated operative note. The type of prior repair, presence of mesh, and proximity of suture to nerves were noted. A uniform pain scale was not utilized and therefore pain data cannot be obtained. These were elective recurrent inguinal hernia patients with varying degrees of discomfort, not chronic postherniorrhaphy pain patients. Iliohypogastric and genital femoral nerves were selectively resected if suspected of harboring neuritis. It is the bias of this surgeon to routinely perform neurectomy of the ilioinguinal nerve during Lichtenstein repairs.

The suspected nerves were sent for histological examination after planned neurectomy, including a segment in excess of $6 \mathrm{~cm}$. This was examined by the community pathologist and results of the pathologist's reports were tabulated with corresponding operative findings.

Accumulated data of these 30 recurrent inguinal hernia repairs were statistically compared with the results of a similar study of 100 primary inguinal hernia repairs previously published [1]. Whitman University Statisticians compared the data sets.

\section{Results}

Thirty recurrent inguinal hernia operative reports and pathology reports were reviewed and the results were tabulated. Not all nerves were seen during surgery and of the nerves seen the, ilioinguinal was most frequently resected for histologic examination. The genital branch was frequently abnormal if seen, and the iliohypogastric was least frequently abnormal. The presence of neuroma was frequently confirmed by the pathologist (Table 1). These operations were all first time recurrences. Seven of the patients had repair as a minor, but most had prior adult repairs. Eighteen patients had no prior mesh and prior laparoscopic repair was only present in five of the thirty patients (Table 2).

Most of the hernias had been repaired many years prior with only three patients having had repair within the last two years (Table 3 ).

There were 20 patients having inguinal neuritis among thirty recurrent Lichtenstein inguinal hernia repairs (66\%). The most affected nerve in recurrent hernia is the ilioinguinal nerve accounting for $69 \%$ of neuritis cases. Inguinal neuritis most frequently occurred at the site where the nerve emerges from below the external oblique fascia - the external oblique neuroperforatum_-accounting for 69\% in recurrent repair. One patient had neuritis 
Table 1. Thirty recurrent inguinal hernia dissection findings.

\begin{tabular}{ccccc}
\hline & Seen & Not seen & Resected & Neuroma confirmed \\
\hline Iliohypogastric & 13 & 17 & 3 & 3 \\
Ilioinguinal & 23 & 7 & 21 & 18 \\
Genital brach & 6 & 24 & 5 & 5 \\
\hline
\end{tabular}

Table 2. Type of prior inguinal repair.

$\begin{array}{cc}\text { Infant/Child } & 7 \\ \text { Adult (No Mesh) } & 11 \\ \text { Open (Mesh) } & 7 \\ \text { Laparoscopic } & 5 \\ \text { Total } & 30\end{array}$

Table 3. Time interval since prior inguinal hernia repair.

$\begin{array}{cc}\geq 10 \text { Years } & 18 \\ 6 \text { - } 9 \text { Years } & 3 \\ 2 \text { - } 5 \text { Years } & 6 \\ <2 \text { Years } & 3 \\ \text { Total } & 30\end{array}$

associated with earlier placed mesh, but all other instances of neuritis were not associated with materials from prior hernia repair (Table 4).

Iliohypogastric neuritis occurred diffusely in one patient (in association with mesh), and distally at the neuroperforatum in two of three cases (Figure 1).

Ilioinguinal neuritis occurred diffusely in one patient and was in contact with mesh. It was associated with the cord in two patients and at the internal inguinal ring in one patient. In a total of twelve it occurred at the neuroperforatum and in eleven of those this was at the external inguinal ring. Two patients had neuritis evident at a nerve bifurcation point distal to the external inguinal ring (Figure 2).

Neuritis in the genital branch of the genitofemoral nerve occurred at the fascial neuroperforatum in four cases, three of which were at the external inguinal ring. The neuroperforatum in one of these cases though was inferior and lateral to the external inguinal ring. One occurred at a bifurcation point in the medial floor. This was in a female (Figure 3).

A total of 26 nerves were found to have inguinal neuritis, 18 ilioinguinal nerves, 3 iliohypogastric nerves, and 5 with genital branches (Table 1). Neuritis occurred in multiple nerves in recurrent hernia more frequently that found in primary inguinal hernia. Four patients had involvement of both the ilioinguinal and genitofemoral nerves, and two patients had involvement of both the iliohypogastric and the ilioinguinal nerves. There were no patients who had a combination of the genital branch and the iliohypogastric nerve. Overall $20 \%$ of patients with neuritis had multiple nerves involved (Table 5).

Despite the diffuse scarring present in the dissection found in all recurrent patients, neuritis was diffusely evident only in one patient. Focal neuritis was commonly found, and most commonly concentrated at the neuroperforatum (Figure 4). Of 26 nerves exhibiting neuritis, 18 were found at the neuroperforatum, similar to primary inguinal hernia. In five damaged nerves, the site of the neuritis was not described in primary inguinal hernia: two along the spermatic cord, two diffusely (associated with mesh, in the same patient), and one at the internal inguinal ring. Three patients were found to have neuritis occurrence distal to the external inguinal ring associated with nerve bifurcation, as described in primary hernia. 
Table 4. Occurrence of inguinal neuritis in recurrent inguinal hernia.

\begin{tabular}{cc}
\hline Inguinal neuritis & Percent occurrence \\
\hline Incidence & $66 \%$ \\
Ilioinguinal nerve & $69 \%$ \\
Neuroperforatum & $69 \%$ \\
Mesh association & $7.7 \%$ \\
\hline
\end{tabular}

Table 5. Neuritis in multiple nerves with recurrent inguinal hernia.

\begin{tabular}{cc}
\hline & $N=6 / 30(20 \%)$ Neuritis patients \\
\hline Ilioinguinal plus iliohypogastric & 2 \\
Ilioinguinal plus genital branch & 4 \\
Genital branch plus iliohypogastric & 0 \\
\hline
\end{tabular}

Table 6. Inguinal neuritis in recurrent hernia compared with primary inguinal hernia.

\begin{tabular}{cccc}
\hline & Primary hernia $(\mathrm{N}=100)$ & Recurrent hernia $(\mathrm{N}=30)$ & \\
\hline Incidence & $34 \%$ & $66 \%$ & $\mathrm{P}>0.42$ \\
Ilioinguinal & $88 \%$ & $69 \%$ & $\mathrm{P}>0.10$ \\
Neuroperforatum & $83 \%$ & $69 \%$ & $\mathrm{P}>0.36$ \\
Multiple nerves & $1 \%$ & $20 \%$ & $\mathrm{P}<0.01$ \\
\hline
\end{tabular}

\section{Iliohypogastric Neuritis}

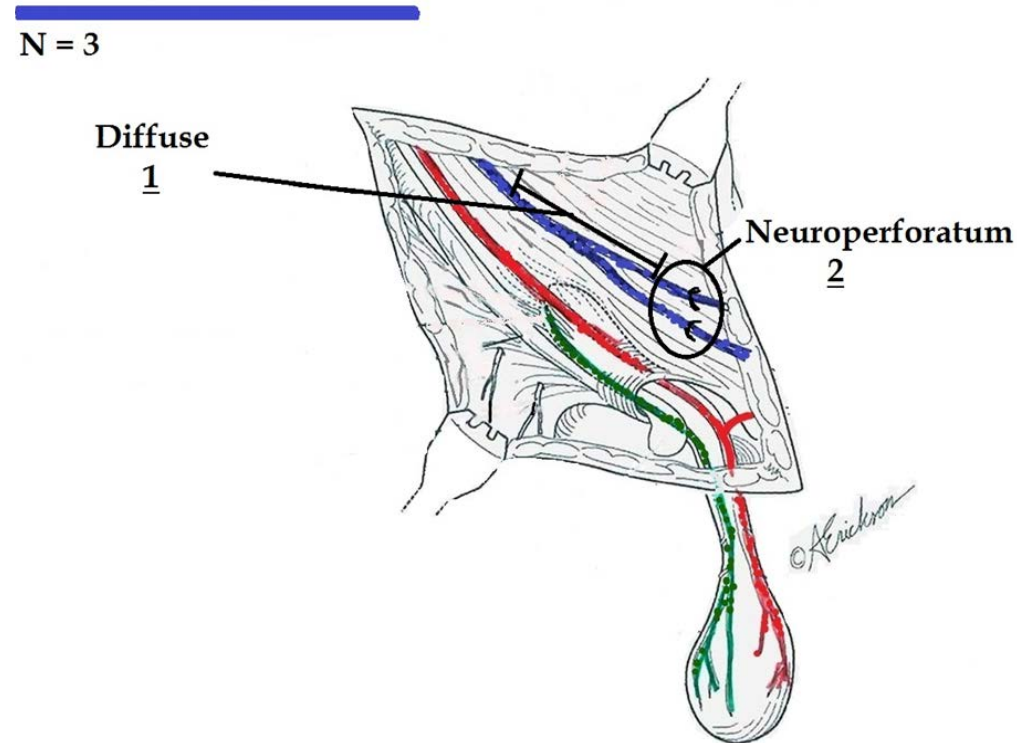

Figure 1. Iliohypogastric neuritis.

Twenty patients were found to have inguinal neuritis among thirty recurrent open inguinal hernia repairs (66\%). This compares to 34\% among primary repairs, but is a similar rate $(\mathrm{P}>0.42)$ assuming the damaged nerve was left intact in 34\% of these recurrences during the primary repair (Table 6).

Two separate nerves were found to exhibit neuritis in six patients (20\%), significantly higher than the $1 \%$ 


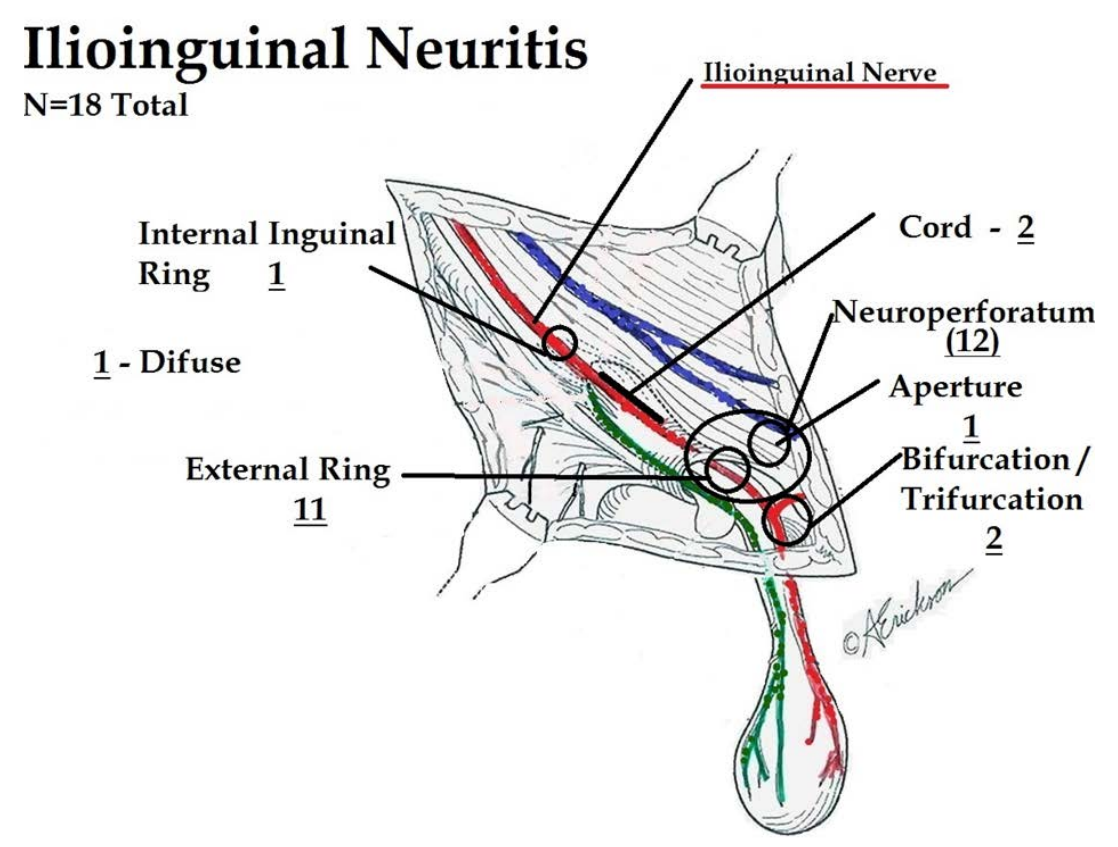

Figure 2. Ilioinguinal neuritis.

\section{Genitofemoral Neuritis}

$$
\mathbf{N}=5
$$

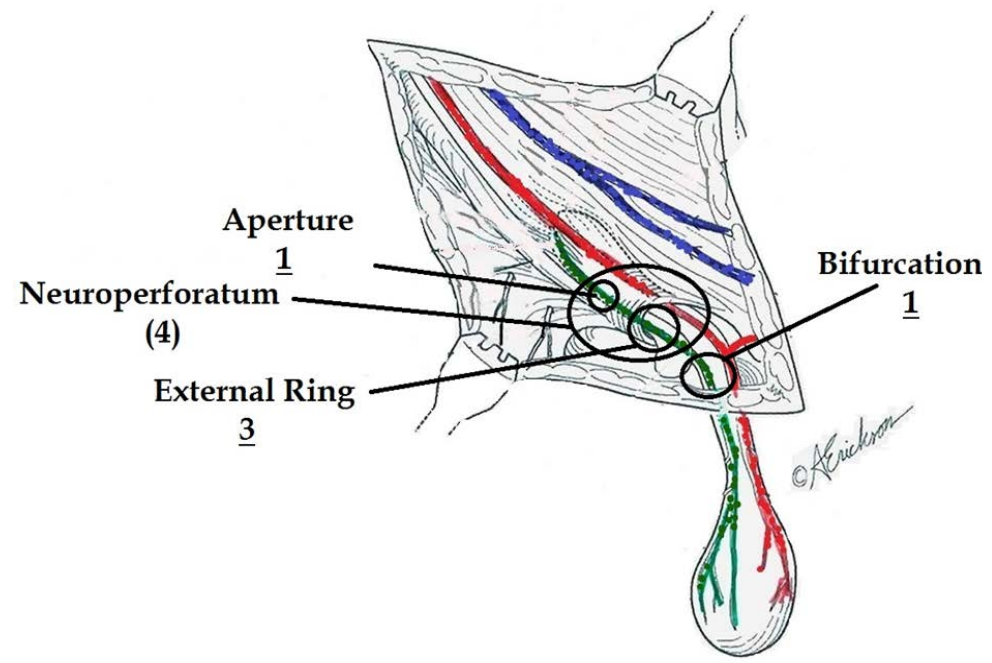

Figure 3. Genitofemoral neuritis.

among primary hernias $(\mathrm{P}<0.01)$ (Table 6).

In recurrent inguinal hernia, $69 \%$ of neuritis occurred in the ilioinguinal nerve compared to $88 \%$ of damaged ilioinguinal nerves in the primary hernia. A test for the difference in proportions gives $\mathrm{P}>0.10$ (Table 6).

The most common site of neuritis in recurrent inguinal hernia was the external oblique neuroperforatum (69\%) compared with $83 \%$ in primary cases. A test for difference in proportion shows $\mathrm{P}>0.36$ (Table 6).

\section{Discussion}

In this study patients underwent elective open repair of recurrent inguinal hernia and had a variety of prior operation types, some of which were performed as children. These were not chronic pain patients but those with 


\section{Inguinal Neuritis by Location}

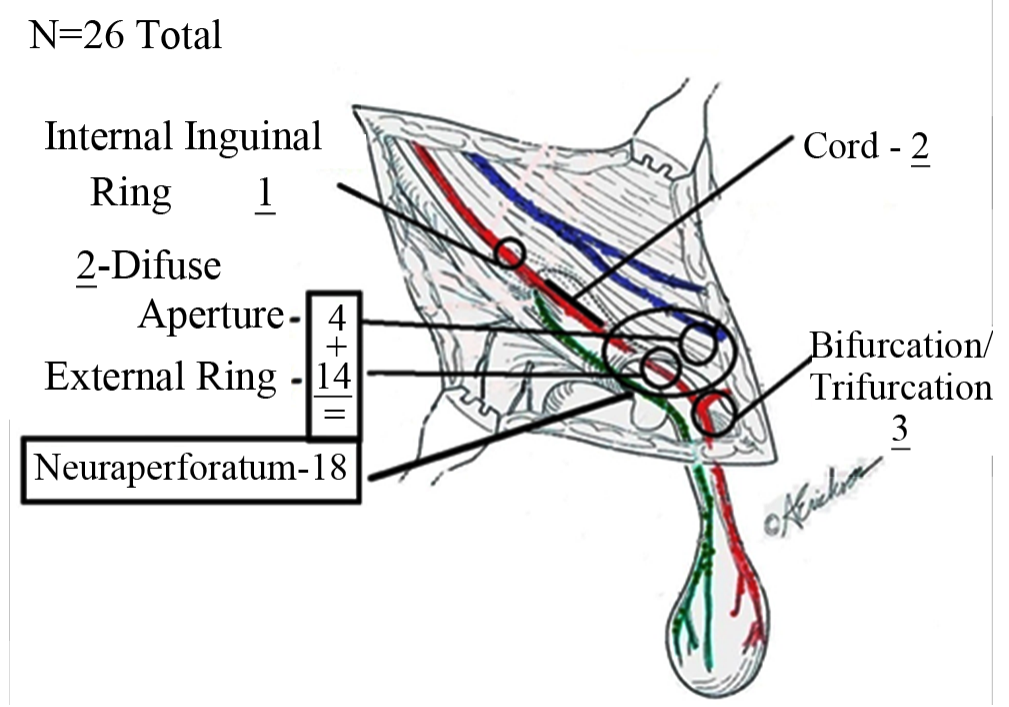

Figure 4. Inguinal neuritis by location.

symptomatic or even asymptomatic hernias. The hypothesis was that prior operative trauma should result in a wider distribution of inguinal neuritis than is present in primary inguinal hernia when comparing primary repair to recurrence repair.

The basic science of histological examination of inguinal hernia nerves is a recent undertaking and may lead to insights into why patients have pain at presentation with their hernia. Numerous studies have shown benefit in routine neurectomy during open inguinal herniorrhaphy [6]-[9]. Neurectomy with open inguinal hernia repair allows for histological examination of nerves previously thought to be normal. This study is not designed to answer pain questions, but to demonstrate sensory nerve damage associated with recurrent inguinal hernia. The first report of sensory nerve damage showed 34\% incidence of inguinal neuritis in a small personal series of 100 consecutive patients of primary inguinal hernia [1]. The nerve was considered a candidate for selective neurectomy in that series if it was found to be thickened to twice the diameter of the nerve within its investing fascia. Typically an abnormal nerve is whitish and adherent to surrounding structures in the primary inguinal hernia. In $88 \%$ of cases this involved the ilioinguinal nerve, and in $93 \%$ of cases the nerve damage occurred within a centimeter of the neuroperforatum - where the nerve pierces the external oblique fascia. Frequently this is at the external inguinal ring; however in $18 \%-28 \%$ of perforation sites with the ilioinguinal nerve this is a small fascial aperture medial to the external inguinal ring [2]-[4]. The iliohypogastric nerve nearly always passes through a similar aperture.

The results, however, demonstrated a strikingly similar distribution of nerve injury with inguinal neuritis occurring in the ilioinguinal nerve in $69 \%$ of patients and at the neuroperforatum in $69 \%$ of patients. It was twice as common however, at $66 \%$. The location of the neuritis suggests that the intraabdominal pressure injures the nerve as it passes along the edge of the external oblique fascia through the neuroperforatum causes histological changes of fibrotic reaction and Renaut body formation. The finding of Renaut bodies has experimentally been shown to be associated with nerve compression sites cadaver studies [10] and experimentally to be caused by compression [11]. This data suggests that primary nerve damage is most frequently caused by the hernia itself, rather than the operative damage.

Inguinal neuritis occurs twice as often in recurrent hernia compared to primary inguinal hernia. Some of this difference may be accounted for by technical differences between the studies. In the primary repair study the ilioinguinal nerve was resected only if suspected of being abnormal, but in recurrent hernia it was routinely resected. Eighteen of twenty one nerves were found histologically abnormal by the pathologist, so if routine neurectomy were carried out in primary repair, the incidence may have been higher than clinically appreciated. Additionally when the recurrent hernias had originally been operated on the surgeon may have left an affected nerve intact only to be discovered after the recurrence. This was assumed to be the case in our statistical analysis, 
but this assumption may not be valid, especially in the repairs done on minors who later became recurrent.

Critics of open herniorrhaphy may point to the higher rate of neuritis in recurrent hernia as evidence that open repair damages nerves. There is some evidence to support this. A single patient with dual nerve neuritis associated with a mesh, a single occurrence at the internal inguinal ring and a higher rate of dual nerve involvement, particularly involving the genital branch. In contradiction six of seven patients with mesh did not have diffuse involvement.

The occurrence of neuritis in the genital branch was uncommon in primary repair (3\%), but higher in recurrent repair (5 of 30,16\%). This may be a small number sampling error of course, but operative factors may be at play. If during the primary repair the spermatic cord is opened but the cremaster is not closed the genital branch can be directly exposed to scarring or mesh and consequently the direct frictional forces at the external inguinal ring (3 of 5 cases of genital neuritis). Closure of the cremaster is now recommended by Ahmed to prevent this type of nerve exposure. Similarly, neuritis was found in a woman whose round ligament was preserved rather than resected during her primary repair; thus leaving the genital branch exposed.

In this study inguinal neuritis occurred in multiple nerves in 20\% of patient's undergoing recurrent inguinal hernia repair; this seems a departure from the findings in primary inguinal hernia repair. For surgeons undertaking open herniorrhaphy it is recommended these nerves routinely be examined at their full length as the site of involvement is not always as uniform as with primary herniorrhaphy. Additionally the occurrence of neuritis in the genital branch, if not protected within the cremaster muscle, is significantly higher among recurrent inguinal hernia patients and should be watched for carefully.

The general consensus permeating literature regarding postherniorrhaphy pain is that the surgeon, by whatever technique he has used, has actively caused damage to the nerves [12]-[16]. The Reinpold study [17] has now shown that mobilization of the ilioinguinal nerve during open repair causes short term and long term pain with data extending out to ten years. In patients with chronic postherniorrhaphy pain the patient's reoperation to alleviate that pain many times shows evidence of direct injury from suture, tacks, and mesh contraction [18]. In this series, although nerves were abnormal in 66\%, only one patient had neuritis associated with foreign body.

The preponderance of neuritis cases however, occur in a similar distribution as found in primary herniorrhaphy. Namely the ilioinguinal nerve involvement at the neuroperforatum. This suggests that force vectors common to the hernia are inflicting damage to nerves in its trajectory as the hernia descends within the inguinal canal. This is clinically seen as the mushrooming of the hernia seen near the external inguinal ring.

The primary hernia patient is most frequently motivated to proceed with repair because of pain. The question of why hernia pain is present may lie in the histology. Recent autopsy studies in incidental hernia have garnered several destructive findings near the internal inguinal ring including arterial intimal hyperplasia and occlusion, venous engorgement [19], and degeneration of muscle and motor nerves [20]. Additionally the findings of sensory nerve deterioration due to mechanical effects of the hernia may predispose to neurogenic pain preoperatively, as well as postoperatively in preserved nerves.

Studies have demonstrated that preoperative pain strongly correlates with chronic postherniorrhaphy pain [21]-[24]. Since histology demonstrates frequent pathology in sensory nerves, the local compressive effect of the hernia may have already set the course for chronic pain syndrome. Both O’Dwyer [25] and Fitzgibbons [26] have now concluded that most patients with watchful waiting will convert to operative course within ten years, predominantly for reasons of pain. It is therefore advisable to operate on patients with asymptomatic hernia before neuritis or other permanent degenerative changes can become a part of the pathology in the inguinal canal.

The utility of this study is limited by a number of factors. The primary purpose of these patient's operations was to repair the hernia not to conduct a thorough anatomical dissection. Therefore the iliohypogastric nerve was not seen in 17 of 30 patients. Therefore the incidence of iliohypogastric neuroma may be underestimated. Secondly the genital branch was usually not seen and also may be underrepresented. The ilioinguinal nerves were not identifiable in 7 of 30 patients also indicating there may be a gross underestimation of the incidence of neuroma in these specimens. Many surgeons in the past had not admitted to resecting the ilioinguinal nerve but were routinely doing it and it is possible the ilioinguinal nerve may have been removed in some of these patients with the prior repair.

Most inguinal hernia repairs performed in the open fashion now are performed with mesh. Seventeen of these patients had not been repaired with mesh. The finding of dual nerve involvement with one of the seven open mesh patients would suggest that there may be more diffuse nerve involvement in patients with routine utilization of mesh. This has certainly been suggested theoretically by those who prefer a laparoscopic positioning of 
mesh away from the nerves. The small sample size with mesh does not allow a good evaluation of that possibility.

This is a small introductory study with limited findings and further research must be conducted to find if any correlation with preoperative pain exists. Preoperative pain scale studies correlating inguinal neuritis need to be routinely applied to patients undergoing neurectomy. Resected nerves should be consistently submitted for histological examination with attention to the thickened segment.

\section{Conclusions}

The overall incidence of inguinal neuritis was $66 \%$ in recurrent inguinal hernia repairs. The ilioinguinal nerve was most commonly affected in these recurrent hernias. Inguinal neuritis occurs more commonly in recurrent inguinal hernia repair compared with primary inguinal hernia; nevertheless, it has essentially the same distribution when it comes to the ilioinguinal nerve.

With recurrent hernia however, involvement of more than one nerves and involvement of the genital branch are more common and the entire operative field should be examined very closely for evidence of neuritis in recurrent inguinal hernias. The hypothesis that inguinal neuritis will be found in more nerves and widely distributed locations of neuritis is supported by these data.

\section{Acknowledgements}

Pathology: Eric Arntson, M.D.; Larry O’Bryant, M.D.; Don Born, M.D., PhD. Statistics: David DeVine, Whitman University.

\section{References}

[1] Wright, R., Sanders, E., et al. (2011) Inguinal Neuritis is Common in Primary Inguinal Hernia. Hernia, 15, 393-398. http://dx.doi.org/10.1007/s10029-011-0807-z

[2] Ndiaye, A., Diop, M., et al. (2007) Anatomical Basis of Neuropathies and Damage to the Ilioinguinal Nerve during Repair of Groin Hernias (about 100 Dissections). Surgical and Radiologic Anatomy, 29, 675-681. http://dx.doi.org/10.1007/s00276-007-0272-7

[3] Mandelkov, H. and Loeweneck, H. (1988) The Iliohypogastric and the Ilioinguinal Nerves. Surgical and Radiologic Anatomy, 10, 145-149. http://dx.doi.org/10.1007/BF02307823

[4] Roberts, W., Engen, P., et al. (1983) A Proposed Method of Dissection of the Anterolateral Abdominal Wall—Applied Features. Anatomischer Anzeiger, 154, 111-117.

[5] Ferzli, G., Edwards, E., et al. (2008) Postherniorrhaphy Groin Pain and How to Avoid It. Surgical Clinics of North America, 88, 203-216. http://dx.doi.org/10.1016/j.suc.2007.10.006

[6] Mui, W., Ng, C., et al. (2006) Prophylactic Ilioinguinal Neurectomy in Open Inguinal Hernia Repair-A Double Blind Randomized Controlled Trial. Annals of Surgery, 244, 27-33. http://dx.doi.org/10.1097/01.sla.0000217691.81562.7e

[7] Malekpour, F., Mirhashemi, S., et al. (2008) Ilioinguinal Nerve Excision in Open Mesh Repair of Inguinal HerniaResults of a Randomized Clinical Trial; Simple Solution for a Difficult Problem? The American Journal of Surgery, 195, 735-740. http://dx.doi.org/10.1016/j.amjsurg.2007.09.037

[8] Johner, A., Faulds, J. and Wiseman, S. (2011) Planned Ilioinguinal Nerve Excision for Prevention of Chronic Pain after Inguinal Hernia Repair: A Meta-Analysis. Surgery, 150, 534-541. http://dx.doi.org/10.1016/j.surg.2011.02.024

[9] Karakayali, F., Oksuz, E., et al. (2010) Effectiveness of Multiple Neurectomies to Prevent Chronic Pain after Tension-Free Hernia Repair. International Surgery, 95, 40-48.

[10] Jefferson, D., Neary, D. and Eames, R. (1980) Renaut Body Distribution at Sites of Human Peripheral Nerve Entrapment. Journal of the Neurological Sciences, 49, 19-29. http://dx.doi.org/10.1016/0022-510X(81)90184-2

[11] Ortman, J., Sahenk, Z. and Mendel, J. (1983) The Experimental Production of Renaut Bodies. Journal of the Neurological Sciences, 62, 233-241. http://dx.doi.org/10.1016/0022-510X(83)90202-2

[12] Ferzli, G., Edwards, E., et al. (2008) Postherniorrhaphy Groin Pain and How to Avoid It. Surgical Clinics of North America, 88, 203-206. http://dx.doi.org/10.1016/j.suc.2007.10.006

[13] Aasvange, E. and Kehlet, H. (2005) Chronic Postoperative Pain: The Case of Inguinal Herniorrhaphy. British Journal Anesthesiologist (Brajan Anesth), 95, 69-76. http://dx.doi.org/10.1093/bja/aei019

[14] Franneby, U., Sandblom, G., et al. (2006) Risk Factors for Long Term Pain after Hernia Surgery. Annals of Surgery, 244, 212-219. http://dx.doi.org/10.1097/01.sla.0000218081.53940.01 
[15] Linderoth, G. and Kehlet, H. (2011) Neurophysiological Characteristic of Persistent Pain after Laparoscopic Inguinal Hernia Repair. Hernia, 15, 521-529. http://dx.doi.org/10.1007/s10029-011-0815-z

[16] Kehlet, H., Curlick, H., Jensen, T., et al. (2006) Persistent Postsurgical Pain: Risk Factors and Prevention. Lancet, 367, 1618-1625. http://dx.doi.org/10.1016/S0140-6736(06)68700-X

[17] Reinpold, W., Nels, J. and Eggert, A. (2011) Nerve Management and Chronic Pain after Open Inguinal Hernia Repair -A Prospective Two Phase Study. Annals of Surgery, 254, 163-168. http://dx.doi.org/10.1097/SLA.0b013e31821d4a2d

[18] Amid, P. (2011) Surgical Treatment of Postherniorrhaphy Chronic Pain. Hernia, 15, 239-249.

[19] Amoto, G. and Romano, G. (2012) Damage to Vascular Structures in Inguinal Hernia Specimens. Hernia, 16 , 63-67. http://dx.doi.org/10.1007/s10029-011-0847-4

[20] Amato, G., Ober, E., et al. (2011) Nerves Generation in Inguinal Hernia Specimens. Hernia, 15, 53-58. http://dx.doi.org/10.1007/s10029-010-0735-3

[21] O’Dwyre, P., Alani, A., McConnachie, A., et al. (2005) Groin Hernia Repair: Postherniorrhaphy Pain. World Journal of Surgery, 29, 1062-1065. http://dx.doi.org/10.1007/s00268-005-7903-0

[22] Franneby, U., Sandblom, G., et al. (2006) Risk Factors for Long-Term Pain after Hernia Surgery. Annals of Surgery, 244, 212-219. http://dx.doi.org/10.1097/01.sla.0000218081.53940.01

[23] Wright, D., Patterson, C., Scott, N., et al. (2002) Five Year Follow-Up of Patients Undergoing Laparoscopic or Open Groin Hernia Repair: A Controlled, Randomized Trial. Annals of Surgery, 235, 333-337. http://dx.doi.org/10.1097/00000658-200203000-00004

[24] Poobalanas, B.J., King, P.M., et al. (2001) Chronic Pain and Quality of Life Following Open Hernia Inguinal Repair. British Journal of Surgery, 88, 1122-1126. http://dx.doi.org/10.1046/j.0007-1323.2001.01828.x

[25] Chung, L., Norrie, J. and O’Dwyer, P. (2011) Long-Term Follow-Up of Patients with Painless Inguinal Hernia from a Randomized Clinical Trial. British Journal of Surgery, 98, 596-599. http://dx.doi.org/10.1002/bjs.7355

[26] Fitzgibbons, R., Ramanan, B., et al. (2013) Long-Term Results of a Randomized Controlled Trial of Nonoperative Strategy (Watchful Waiting) for Men with Minimally Symptomatic Inguinal Hernias. Annals of Surgery, 258, 508-515. http://dx.doi.org/10.1097/SLA.0b013e3182a19725 
Scientific Research Publishing (SCIRP) is one of the largest Open Access journal publishers. It is currently publishing more than 200 open access, online, peer-reviewed journals covering a wide range of academic disciplines. SCIRP serves the worldwide academic communities and contributes to the progress and application of science with its publication.

Other selected journals from SCIRP are listed as below. Submit your manuscript to us via either submit@scirp.org or Online Submission Portal.
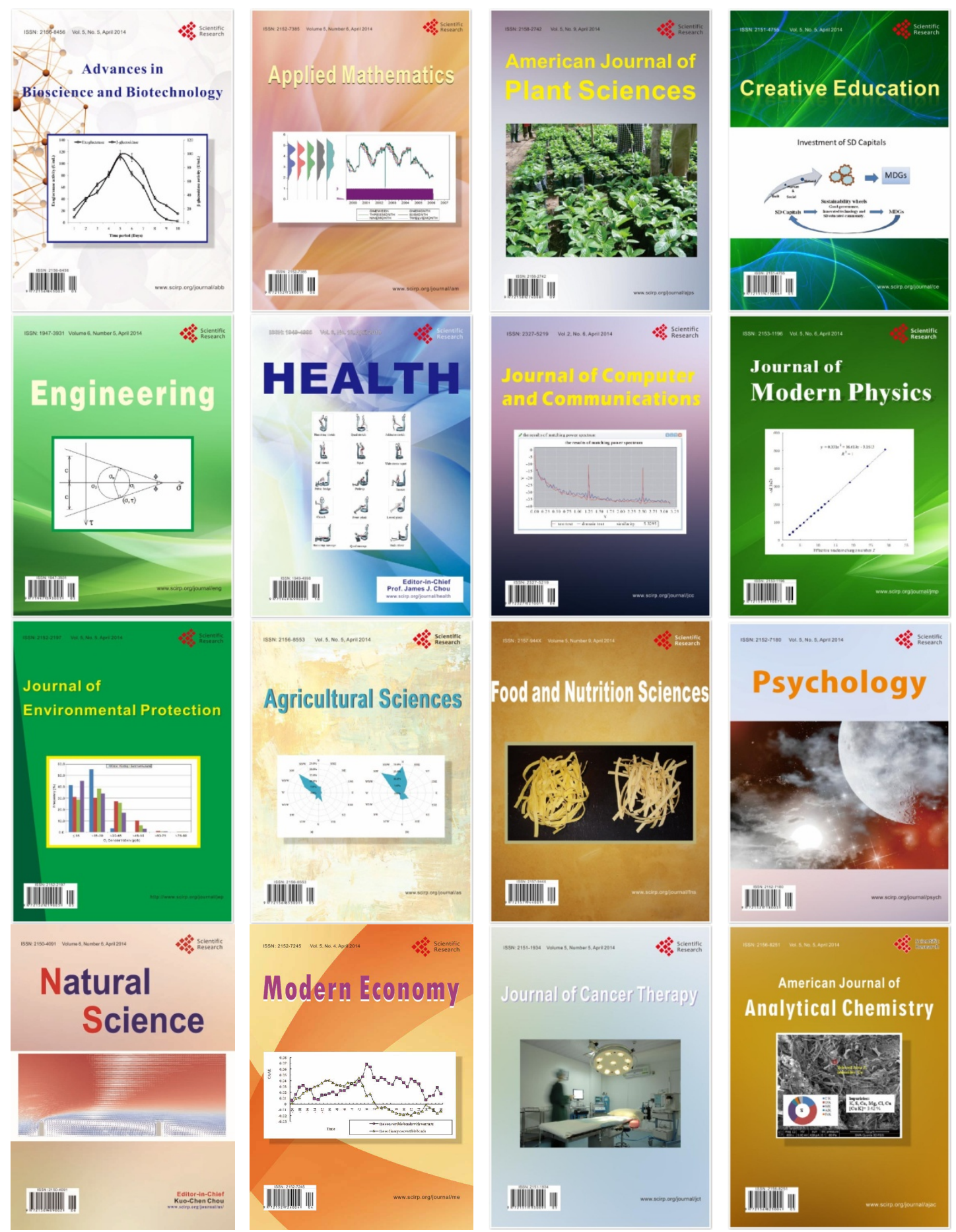